\title{
Site selection and characterization for giant telescopes
}

\section{Matthias Schock}

Matthias Schock, "Site selection and characterization for giant telescopes," Proc. SPIE 5489, Ground-based Telescopes, (28 September 2004); doi: $10.1117 / 12.551847$

EDent: SPIE Astronomical Telescopes + Instrumentation, 2004, Glasgow, United Kingdom 


\title{
Site selection and characterization for giant telescopes
}

\author{
Matthias Schöck \\ California Institute of Technology, Pasadena, CA, USA
}

\begin{abstract}
I describe the procedures used during the different phases of site testing for astronomical telescopes, that is, during the pre-selection of candidate sites, on-site testing (site selection), the site decision itself and characterization of the telescope site once the decision has been made. Many of the important parameters for astronomical night-time telescopes and some of the methods that can be used to determine these parameters are described. There appear to be no fundamental differences between the methods used for telescopes in the 5 to 10 meter range and telescopes with diameters larger than 10 meters, although specific differences certainly exist.
\end{abstract}

Keywords: Site testing, site selection, extremely large telescopes

\section{INTRODUCTION}

Site testing and selection are among the critical steps in the early stages of an astronomical telescope project and site characterization continues after the site decision has been made. In this paper, I describe the steps that are (or can be) taken during a typical telescope project.* The main emphasis is on the differences between site selection procedures for the coming generation of telescopes with mirror diameters of several tens of meters hereafter referred to as giant telescopes - and the previous generation of telescopes in the 5 to 10 meter class (large telescopes). The emphasis of this paper is on night-time telescopes, although many concerns equally apply to solar observatories.

Site testing for an astronomical telescopes generally follows a three-stage pattern. The first stage is the pre-selection of a shortlist of candidate sites. During the second stage, these candidate sites are tested in detail, usually by deploying on-site equipment. In this paper, I refer to the second stage as the site selection stage. It is concluded by the actual selection and acquisition of the site on which the telescope is to be built. The last stage, called site characterization in the framework of this paper, is a more in-depth study of the telescope site which can extend though the final design phase, construction and operation.

There might be variations of this general procedure based on the particular telescope project. For example, one of the three stages might be kept very brief or skipped entirely or there might, at times, not be a clear distinction between two of the stages as they can overlap, both in time and content. One might also consider there to be several stages to site characterization, one for final design, construction and operation each. Nevertheless, most astronomical telescope projects follow roughly the outlined procedure. The main body of this paper is therefore separated into three sections, one for each stage, with the emphasis being on the second stage. As explained above, I pay particular attention to how site testing for a giant telescopes differs from site testing for large telescopes.

\section{PRE-SELECTION OF CANDIDATE SITES}

The first step of a telescope site testing process is always the pre-selection of a shortlist of candidate sites. It is, obviously, not possible to test every mountain or high plateau and every location thereon in detail. Thus, one needs to narrow down the number of sites that could, in theory, be suitable for the telescope. The actual steps taken to arrive at this shortlist vary widely from project to project. Some possibilities are as follows:

\footnotetext{
*In spite of the author's affiliation with the Thirty Meter Telescope, this paper is meant to be a general description of site testing for extremely large telescopes and not to concentrate on a particular project. It will, however, unavoidably be biased by the philosophy followed in the TMT site selection process. For an outline of the current status and strategy of TMT site selection see Ref. 1 .
} 
Conventional wisdom: Astronomers have been building telescopes for decades, even centuries by now and it is known that there are two types of sites which usually provide good or superb conditions in terms of atmospheric properties. The first type is an isolated high mountain on islands in temperate oceans, where the weather is good and a laminar air flow and large thermal inertia of the surrounding ocean keep the inversion layer low. This type of sites includes Mauna Kea and La Palma. The second type contains sites in coastal mountain ranges with a cold ocean upwind providing a laminar airflow and, again, a low inversion layer. These conditions exist on the coasts of California, Baja California, Chile and Namibia.

It is generally believed that continental sites are inferior to these two types of sites, mainly due to the turbulence-generating topography, but there are exceptions to this rule. An example is Maidanak in Uzbekistan, ${ }^{2}$ which has been shown to have very good seeing.

Former site studies, existing observatories: It is obvious that results from former site studies and information available from existing observatory sites can (and should) be used to help with the pre-selection of sites.

Remote studies: Satellite studies of cloud cover and precipitable water vapor (PWV) can also be used to aid in the pre-selection of candidate sites. These studies provide estimates of the percentage of clear (photometric and usable) nights and the PWV distributions of sites. This method has been studied and used by the European Southern Observatory $(\mathrm{ESO})^{3}$ and has been used to help with the pre-selection of sites for the Thirty Meter Telescope (TMT). ${ }^{4}$ Climatology studies ${ }^{5}$ are also sometimes used, to assess the long-term properties of a site.

Modeling: Air flow and turbulence as well as some meteorological parameters over a site can be simulated using theoretical models. Such simulations include, for example, computational fluids dynamics ${ }^{6}$ and mesoscale models. ${ }^{7,8}$ Usually, models are not used to produce the first (long) shortlist of sites - although this is, in principle, possible - but rather to narrow down the initial shortlist or to decide on a particular location on a given mountain if the topography is not straight forward.

Other concerns and issues: There are also reasons to exclude sites from the shortlist, or include them in it, that have little to do with the atmospheric properties of a site. A site might simply be impractical from a logistical point of view (access, cost of construction or operation, etc.) to be considered seriously or the proximity of a close town or mine might make operation of an astronomical telescope impossible due to the light pollution, smog or dust it produces. A list of considerations going into the site decision are given in the following section. Many of them also influence the pre-selection process.

It might also not be clear a priori whether a specific site or a certain location on a mountain is preferable to another and there might not be sufficient resources to test or model all possible locations. In this case, one might simply have to make a (hopefully educated) guess and go from there.

In most projects, the process at which one arrives at a shortlist of candidate sites is a combination of the procedures described above. One might use one method to get a long shortlist and then another to narrow it down to the list of sites that will be studied on-site and/or in more detail. Not every project goes through all of these steps and the path taken is largely determined by the available time, manpower and budget. Fundamentally speaking, however, there are no differences between the pre-selection processes used for giant telescopes and large telescopes as one always wants to find the best sites for the given telescope.

\section{ON-SITE TESTING}

The next step is the detailed on-site testing of the sites that came out of the pre-selection process. A list of many of the issues and parameters that need to be considered during this phase is given in Table 1, along with instruments and methods that can be used to determine the parameters. While many of them, of course, already played a role in the pre-selection considerations, they are described in more detail in this section. For completeness, the methods used to arrive at the pre-selection of sites are also listed in the table. Note that this list is by no means complete, neither with respect to the parameters listed nor concerning the methods and instruments. 
Table 1. Table of parameters and issues to be considered during all phases of site testing for astronomical telescopes and of some of the instruments and/or methods that can be used to determine the parameters or address the issues.

\begin{tabular}{|c|c|c|}
\hline Issue & Parameter & Method or Instrument \\
\hline Pre-selection & Site names & $\begin{array}{l}\text { Conventional wisdom } \\
\text { Former site studies } \\
\text { Information from existing observatories } \\
\text { Remote studies } \\
\text { Modeling }\end{array}$ \\
\hline Weather and Sky & $\begin{array}{l}\text { Clear / photometric nights } \\
\text { Precipitable water vapor } \\
\text { Wind speed and direction } \\
\text { Air and ground temperature } \\
\text { Humidity } \\
\text { Barometric pressure } \\
\text { Solar irradiance } \\
\text { Sky brightness } \\
\text { Atmospheric transparency }\end{array}$ & $\begin{array}{l}\text { Turbulence instrument photometry, } \\
\text { allsky camera, satellite data } \\
\text { Radiometers, satellite data } \\
\text { Weather station } \\
\text { Weather station } \\
\text { Weather station } \\
\text { Weather station } \\
\text { Weather station } \\
\text { Imaging, spectroscopy } \\
\text { Imaging, spectroscopy }\end{array}$ \\
\hline Turbulence & $\begin{array}{l}\text { Overall seeing } \\
\text { Turbulence profiles } \\
\text { Isoplanatic angle } \\
\text { Coherence time } \\
\text { Outer scale }\end{array}$ & $\begin{array}{l}\text { DIMM, GSM, many other instruments } \\
\text { MASS, SCIDAR, SODAR, SLODAR, } \\
\text { Balloon/kite/mast-mounted probes, such as } \\
\text { microthermal probes or sonic anemometers, } \\
\text { modeling } \\
\text { Derived from profiles } \\
\text { All of the above to some extent } \\
\text { GSM, (AO systems, interferometers) }\end{array}$ \\
\hline Logistics & $\begin{array}{l}\text { Location, elevation } \\
\text { Ground properties } \\
\text { Construction and operation cost } \\
\text { Site ownership } \\
\text { Accessibility }\end{array}$ & $\begin{array}{l}\text { Maps } \\
\text { Geological studies } \\
\text { Local inquiries, quotes, etc. } \\
\text { Local inquiries } \\
\text { On-site visits }\end{array}$ \\
\hline Miscellaneous & $\begin{array}{l}\text { Dust } \\
\text { Seismicity, volcanoes } \\
\text { Light pollution } \\
\text { Sodium layer properties } \\
\text { Political/social/cultural issues } \\
\text { Environmental issues }\end{array}$ & $\begin{array}{l}\text { Particle sensors, optical methods } \\
\text { Geological studies, accelerometers } \\
\text { Visual inspection, imaging, spectroscopy } \\
\text { LIDAR, spectroscopy } \\
\text { Local inquiries } \\
\text { Local inquiries }\end{array}$ \\
\hline
\end{tabular}

\subsection{Weather and Sky properties}

The first group of parameters is listed in the table as "weather and sky." The interest in the fraction of photometric and clear nights is obvious. It can be addressed by using photometry data from optical turbulence measurement instruments such as a differential image motion monitor (DIMM) ${ }^{9}$ or a multi-aperture scintillation sensor (MASS),$^{10}$ with the disadvantage that these instruments are restricted to line-of-sight measurements. Deploying allsky cameras produces a more complete picture. In addition, satellite and climatology studies can be used in the pre-selection phase or to investigate the cloud behavior over longer periods of time than that available for on-site testing. Precipitable water vapor (PWV) can also be estimated from satellite data or can be measured on-site using tipping radiometers.

Standard weather parameters like temperatures, humidity, barometric pressure and solar irradiance are of interest mostly for telescope operational purposes. On the other hand, average and maximum wind speeds 
might be extremely important for structural design purposes. All these parameters can be measured easily using commercially available weather stations. It might be of interest to measure such parameters at several elevations above the ground.

Sky properties such as sky brightness and atmospheric transparency can also be important, depending on the purpose of the telescope. They can, in principle, be measured with allsky cameras, although this is much more difficult that assessing the cloud cover. Other kinds of imaging or spectroscopic methods might also be used.

There is no fundamental dependence on the telescope size in the procedures used to measure any of the parameters listed in this section, although some of them might be of greater importance for a larger telescope. For example, the wind load on the telescope structure becomes more important as the telescope size grows. On the other hand, PWV might be more of an issue for a telescope that is used for mid-infrared observations than for visible and near-infrared observations, which is, at least to first order, not dependent on the telescope size. Thus, one can see that the differences between telescopes of different size are analogous to those between telescopes of the same size but with different purposes. They are not fundamental as far as the methods used are concerned, but rather in how the limiting values for parameters are set or how different parameters are weighted in the site decision.

\subsection{Turbulence}

A large fraction of the on-site testing effort is usually dedicated to turbulence measurements. This is due to the importance of the seeing for large telescopes operating at visible and near-infrared wavelengths and to the fact that it is more difficult to measure turbulence reliably than many of the other parameters of interest. Arguably the most important turbulence parameter is the overall seeing angle. There are many instruments that can measure the seeing, such as the Generalized Seeing Monitor (GSM) ${ }^{11}$ and turbulence profilers such as scintillation detection and ranging (SCIDAR) instruments. ${ }^{12}$ The most commonly used instruments for this purpose are DIMMs because of the simplicity and the robustness of the method.

Turbulence profiles are of interest for several reasons. For example, they affect the choice and design of adaptive optics (AO) systems and might aid with the choice of a particular location on a given site if the topography is not simple. Usually, the overall seeing can also not be measured from the same height above the ground at which the primary mirror of the telescope will be located. Measuring the low-altitude turbulence profile then helps to assess whether the seeing measurements need to be corrected for the effects of the ground layer.

There are a multitude of methods that can be used for measuring different parts of the turbulence profile and only some of them are mentioned here. Scintillation-based methods, of which the MASS and generalized SCIDAR instruments are examples, can, in principle, measure the entire turbulence profile, although not every instrument can necessarily access the entire profile and resolution is low for some instruments. The slope detection and ranging (SLODAR) method ${ }^{13}$ uses wavefront sensors and measures the wavefront slope of light from binary stars. Acoustic instrument such as SODARs (sound detecting and ranging) ${ }^{14}$ can produce high-resolution profiles from a few tens to a few hundreds of meters, or even up to a couple kilometers above the ground. Thinwire instruments such as microthermal probes or thermocouples ${ }^{15}$ can be mounted on masts and be suspended from kites or balloons to take in-situ measurements of temperature fluctuations $\left(C_{T}^{2}\right)$ which can then be converted to $C_{N}^{2}$ values. Sonic anemometers can also be used, mounted on masts, to measure $C_{T}^{2} \cdot{ }^{16} \mathrm{~A}$ number of turbulence parameters can be derived from turbulence profiles of which the isoplanatic angle is given as an example in the table.

Two more turbulence parameters of interest are the coherence time and the outer scale of turbulence, $L_{0}$. The coherence time is of great importance for design and operation of $\mathrm{AO}$ systems. The outer scale also has an effect on adaptive optics design and operation. If the telescope is large enough, a small $L_{0}$ can also significantly reduce the uncorrected image width with respect to turbulence of the same coherence length, $r_{0}$, and large $L_{0}$. Most of the methods listed above produce some kind of coherence time measurement, although the conversion to a coherence time useful for AO design might not be trivial. The outer scale is difficult to measure and only one compact turbulence monitor exists which routinely measures $L_{0}$, the GSM. The GSM is, however, not suited for prolonged operation at remote sites. Other methods to measure the outer scale, such as using AO systems 
on large telescopes or telescope interferometers ${ }^{17}$ are obviously not feasible for site testing. The outer scale is therefore usually not measured in telescope site selection other than during short campaigns.

As with the weather and sky parameters, there is no fundamental difference in the methods and instruments used for giant or large telescope site selection. Some of the parameters, such at the outer scale or the precision with which the parameters are measured might be more important for a larger telescope. Also, the budget available for site selection might be larger for a larger telescope so that more detailed studies can be performed. But as before, these are not fundamental differences of the procedures but rather specific difference in the applications of the methods.

\subsection{Logistics}

Logistical concerns affect all phases of a telescope project, from site selection to construction to operation. Things to consider include the location and altitude of the site, access, ground properties and site ownership. They determine how difficult it is to test and develop the site and, together with consideration like material and labor cost, can be used to determine construction and operation costs. Most logistical issues are relatively straightfoward to address and are often already partially considered during the pre-selection phase.

\subsection{Miscellaneous Issues}

There is a large number of other concerns playing a role in the selection of a telescope site. Some are listed under "miscellaneous" in Table 1. Dust close to the ground plays a role both for telescope operation and maintenance, while higher-altitude dust has an affect on atmospheric transparency. Low-elevation dust can be measured using particle sensors. High-elevation dust is detected by (some of) the methods used to measure atmospheric transparency.

Earthquakes are an obvious problem for large structures such as telescopes and the surrounding buildings and nearby volcanoes can cause problems both by the related earthquakes and the ash thrown out in an eruption. The earthquake and volcano risk can be addressed in geological studies and the current activity can be measured using, for example, accelerometers. With growing population and/or technological "improvements" almost anywhere, light pollution is a concern at most existing and potential observatory sites (see, for example, the story of Mt. Wilson in Ref. 18). It can be assessed using visual inspections and imaging and spectroscopic methods. In many places, astronomers are in contact with the local authorities to minimize or reduce light pollution. As a final example, sodium layer properties are of importance for telescopes envisioning to use laser guide star AO systems. The thickness and altitude (and variations thereof) of the sodium layer can be measured with methods such as LIDAR (light detection and ranging). ${ }^{19}$

Finally, political, social and cultural issues are different for each potential telescope site and need to be addressed at all stages of a telescope project. In many cases it is also necessary to perform studies in order to minimize the impact on the environment.

Differences in the methods listed here are, again, not fundamental. Some parameters such as seismicity or sodium layer properties might play a larger role for a larger telescope, but these are, as before, rather differences in the application of the methods and the exact weighting of the individual parameters and not differences in the procedures themselves.

\section{SITE DECISION}

At the end of the site selection phase, the telescope site decision is made. In most cases, this is a highly non-trivial task. Unless the telescope has a specific purpose or there are other reasons that makes one of the site parameters much more important that most or all others, there are usually many factors to be taken into account which cannot usually all be optimized simultaneously. For example, in northern Chile sites close to the coast usually have a higher fraction of clear nights, while inland sites are significantly higher in altitude, which means lower PWV. In a case like this it needs to be decided whether the scientific advantage of the drier site justifies a loss of some clear nights and the extra effort and cost caused by the altitude. A similar case might exist if one site has, for example, better seeing but is located in a seismically more active zone than another site. The site decision generally involves the consideration of many trade-offs like these. 
There are obvious differences between telescope projects concerning how the actual site decision is made. The differences originate from the specific purpose of the telescope (science to be accomplished, general-purpose versus specialized telescope, etc.) but often just as much from practical concerns, such as the budget or the ease/difficulty of construction and operation. The size of the telescope plays a role in that certain parameters might be more or less important for larger telescopes and simply through the fact that the number of telescope that will eventually exist will be smaller as the telescope size gets larger. However, this is again not a fundamental difference in the procedures used to select telescopes of different sizes, but rather in the specific choice of the parameters and their weighting functions.

\section{SITE CHARACTERIZATION}

Site testing for most telescopes does not stop the moment the telescope site has been chosen. Instead, one often concentrates one's efforts on the selected site. The result is first of all a longer temporal baseline of measurements for the site to verify that the data collected during the site selection phase are indeed representative for the site. Furthermore, it is possible to concentrate instruments and efforts that were before spread out over several sites in one location. This can be used to distinguish between individual locations on the same mountain or to access parameters that were not accessible with a smaller instrument suite. An example of such a parameter space extension is the combination of several DIMM telescopes to measure the outer scale of turbulence in the fashion of the Generalized Seeing Monitor, GSM. Finally, it might also be possible to add other instruments that were simply to expensive or too work intensive to operate on several sites simultaneously during the site selection phase.

Just as with all previous phases, there is no fundamental dependence of the methods used on the telescope size. The specific path chosen, how much site characterization is done (and if at all), what parameters are measured and for how long, etc. depends, of course, on the particular project, but given the same budget and the same telescope purpose, the methods and instruments used would likely be very similar.

\section{CONCLUSIONS}

I have outlined the general procedures used during the phases of site testing for astronomical telescopes, that is, during the pre-selection of candidate sites, on-site testing (site selection), the site decision itself and characterization of the telescope site once the decision has been made. I described many of the important parameters for astronomical night-time telescopes and outlined some of the methods that can be used to determine these parameters.

There does not appear to be a dependence on the size of the telescope (assuming that it is at least several meters in diameter so that turbulence does matter) as far as the procedures to be followed are concerned. Differences originate rather from the specific purpose of the telescope, practical concerns and the available resources. A dependence on size does exist in that certain parameters or effects (for example, the outer scale of turbulence and mechanical problems due to wind shake or earthquakes) might become more important for a larger telescope than they are for smaller telescopes and that there will likely be fewer giant telescopes than large telescopes. These differences do, however, simply affect the weight that is given to individual parameters during the site testing phases and is, in general, not per se distinguishable from differences such as those caused by differences in science requirements between telescopes of the same size.

A remaining difference is that, due to its higher cost, a larger telescope often has a larger budget for site testing than a smaller telescope. A more intensive site testing program can then be executed. For the same reasons, it is also possible to make a "more expensive mistake," not just in terms of resources, but also because there will be fewer other telescopes of the same size that could be located at another site to cover science potentials missed due to the given telescope's location.

\section{ACKNOWLEDGMENTS}

The Thirty Meter Telescope (TMT) Project is a partnership of the Association of Universities for Research in Astronomy (AURA), the Association of Canadian Universities for Research in Astronomy (ACURA), the California Institute of Technology and the University of California. The partners gratefully acknowledge the support 
of the Gordon and Betty Moore Foundation, the US National Science Foundation, the National Research Council of Canada, the Natural Sciences and Engineering Research Council of Canada, and the Gemini Partnership.

\section{REFERENCES}

1. W. Skidmore, M. Schöck, A. Tokovinin, S. G. Djorgovski, A. Walker, R. Blum, T. Travouillon, J. Seguel, E. Bustos, D. Walker, J. Vasquez, and P. Gillett, "The Thirty Meter Telescope site testing system," in Ground-based Telescopes, J. M. Oschmann and M. Tarenghi, eds., Proc. SPIE 5489, 2004.

2. S. A. Ehgamberdiev, A. K. Baijumanov, S. P. Ilyasov, M. Sarazin, Y. A. Tillayev, A. A. Tokovinin, and A. Ziad, "The astroclimate of Maidanak Observatory in Uzbekistan," Astron. Astrophys. Suppl. Ser. 145, pp. 293-304, Aug. 2000.

3. See the ESO astroclimatology website at http://www.eso.org/gen-fac/pubs/astclim/forecast/erasmus/.

4. M. Schoeck, D. A. Erasmus, S. G. Djorgovski, G. A. Chanan, and J. E. Nelson, "CELT site testing program," in Future Giant Telescopes, J. R. P. Angel and R. Gilmozzi, eds., Proc. SPIE 4840, pp. 541-552, Jan. 2003.

5. B. M. Garcia-Lorenzo, J. J. Fuensalida, E. G. Mendizabal, C. Muñoz-Tuñon, and A. Varela, "Climatology databases as a tool for the ELT site selection," in Ground-based Telescopes, J. M. Oschmann and M. Tarenghi, eds., Proc. SPIE 5489, 2004.

6. K. Vogiatzis and D. Hiriart, "Numerical wind modeling for the San Pedro Mártir Sierra in Baja California," Revista Mexicana de Astronomia y Astrofisica 40, pp. 81-98, Apr. 2004.

7. E. Masciadri, R. Avila, L. J. Sánchez, S. Cuevas, F. Garfias, A. Agabi, M. Azouit, and J. Vernin, "3D optical turbulence characterization at San Pedro Mártir," in Revista Mexicana de Astronomia y Astrofisica Conference Series, pp. 63-71, Sept. 2003.

8. See the ESO astroclimatology website at http://www.eso.org/gen-fac/pubs/astclim/forecast/mm5/.

9. M. Sarazin and F. Roddier, "The ESO differential image motion monitor," Astron. Astrophys. 270, pp. 294300, 1990.

10. V. Kornilov, A. A. Tokovinin, O. Vozyakova, A. Zaitsev, N. Shatsky, S. F. Potanin, and M. S. Sarazin, "MASS: a monitor of the vertical turbulence distribution," in Adaptive Optical System Technologies II, P. L. Wizinowich and D. Bonaccini, eds., Proc. SPIE 4839, pp. 837-845, Feb. 2003.

11. A. Ziad, R. Conan, A. Tokovinin, F. Martin, and J. Borgnino, "From the Grating Scale Monitor to the Generalized Seeing Monitor," Appl. Opt. 39, pp. 5415-5425, Oct. 2000.

12. A. Fuchs, M. Tallon, and J. Vernin, "Focusing on a turbulent layer: Principle of the Generalized SCIDAR," Pub. Astron. Soc. Pacific 110, pp. 86-91, 1998.

13. R. W. Wilson, "SLODAR: measuring optical turbulence altitude with a Shack-Hartmann wavefront sensor," Mon. Not. R. Astron. Soc. 337, pp. 103-108, Nov. 2002.

14. T. Travouillon, M. C. B. Ashley, M. G. Burton, J. W. V. Storey, and R. F. Loewenstein, "Atmospheric turbulence at the South Pole and its implications for astronomy," Astron. Astrophys. 400, pp. 1163-1172, Mar. 2003.

15. P. Pant and R. Sagar, "Proposed microthermal measurements at Devasthal," Bulletin of the Astronomical Society of India 26, p. 397, 1998.

16. F. Hill, J. Beckers, P. Brandt, M. Collados, C. Denker, J. Kuhn, M. Penn, R. Radick, and K. Streander, "Solar site testing for the Advanced Technology Solar Telescope," in Ground-based Telescopes, J. M. Oschmann and M. Tarenghi, eds., Proc. SPIE 5489, 2004.

17. A. Ziad, M. Schöck, G. A. Chanan, M. Troy, R. Dekany, B. F. Lane, J. Borgnino, and F. Martin, "Comparison of measurements of the outer scale of turbulence by three different techniques," Appl. Opt. 43, pp. 2316-2324, Apr. 2004.

18. R. H. Garstang, "Mount Wilson Observatory: the sad story of light pollution," The Observatory 124, pp. 14-21, Feb. 2004.

19. D. J. Butler, R. I. Davies, H. Fews, R. M. Redfern, N. Ageorges, W. K. Hackenberg, R. Rohloff, S. Rabien, T. Ott, and S. Hippler, "Sodium layer monitoring at Calar Alto by LIDAR," in Adaptive Optical Systems Technology, P. L. Wizinowich, ed., Proc. SPIE 4007, pp. 358-367, July 2000. 